\title{
Hypo-Fractionated Radiotherapy for Laryngeal Cancer; is Linac Based Treatment Better?
}

\author{
Suresh Kumar K. ${ }^{1}$, Anu Asokan ${ }^{2}$ \\ ${ }^{1}$ Department of Radiotherapy, Cancer Care Center, Government Medical College, Kottayam, Kerala, India. \\ ${ }^{2}$ Department of Radiotherapy, Cancer Care Center, Government Medical College, Kottayam, Kerala, India.
}

\section{ABSTRACT}

\section{BACKGROUND}

Carcinoma larynx is the most common malignancy of the upper aero digestive tract. $75 \%$ of larynx cancers are attributable to cigarette smoking and alcohol use. Squamous cell carcinoma constitutes $95 \%$ of all malignant neoplasms of the larynx. Irradiation is the initial treatment for $\mathrm{T} 1$ and $\mathrm{T} 2$ lesions, and surgery is reserved for salvage after radiation therapy failure. Hypo fractionated radiotherapy $(>2.0$ Gy/fraction) has shown comparable local control to conventional radiation in carcinoma Larynx.

\section{METHODS}

A total of 59 patients, newly diagnosed with stage I and II squamous cell carcinoma of glottic larynx who attended the OP clinic of Department of Radiation Oncology, of Kottayam Medical College, from November 2016 to November 2017 who were intended to receive radical treatment with a hypo fractionated regimen of radiotherapy were analysed for local control and toxicity.

\section{RESULTS}

Loco regional control at 1 year of treatment is $93.2 \%$ and Overall survival is $96.61 \%$ and is comparable to published literature data. Majority of the patients in the study had grade 1 - 2 dysphagia, grade 2 - 3 dermatitis, grade 2 - 3 laryngeal mucositis. During $6^{\text {th }}$ week, there was significantly higher incidence of grade 2 and 3 mucositis among patients treated in cobalt 60 machine in 28 out of 31 patients, $(90.3 \%)$ as compared to linear accelerator-based treatment where 17 out of 28 patients $(60.7 \%)$ had grade 2 and 3 mucositis.

\section{CONCLUSIONS}

Hypo fractionated radiotherapy is a safe alternative for conventionally fractionated radiotherapy by reducing overall treatment time by 1 week without affecting locoregional control and survival. It also reduces the socio-economic burden. However linear accelerator-based treatment has better toxicity profile than Cobalt 60 treatment in hypo fractionated radiotherapy for Carcinoma Larynx.

\section{KEY WORDS}

Laryngeal Cancer, Hypo-Fractionation, Radiotherapy, Linear Accelerator
Corresponding Author: Dr. Suresh Kumar K., Additional Professor, Department of Radiotherapy, Cancer Care Center, Government Medical College, Kottayam-686008, Kerala, India. E-mail: drsureshkumar74@gmail.com

DOI: $10.14260 /$ jemds/2019/854

Financial or Other Competing Interests: None.

How to Cite This Article:

Kumar SK, Asokan A. Hypo-fractionated radiotherapy for laryngeal cancer; is linac based treatment better? J. Evolution Med. Dent. Sci. 2019;8(52):3946-3952, DOI: 10.14260/jemds/2019/854

Submission 06-09-2019,

Peer Review 04-12-2019,

Acceptance 10-12-2019,

Published 30-12-2019. 


\section{BACKGROUND}

Carcinoma larynx is the most common malignancy of the upper aero digestive tract, accounting for $1 \%$ of all malignancies and $25 \%$ of head and neck tumours. ${ }^{1} 80 \%$ of larynx cancers occur in men, In India, laryngeal cancer accounts for approximately $3-6 \%$ of all cancers in men. ${ }^{2}$

\section{Aetiology and Pathology}

$75 \%$ of larynx cancers are attributable to cigarette smoking and alcohol use. ${ }^{3}$ Voice abuse, occupational exposure to asbestos, diesel fumes, rubber, and wood dust contribute to the aetiology. 4,5 Deficiency of vitamins- Vitamin C, riboflavin, beta carotene can potentiate carcinogenesis. ${ }^{4,5}$ Squamous cell carcinomas constitutes $95 \%$ of all malignant neoplasms of the larynx. Glottic carcinomas are usually well to moderately differentiated. Carcinomas of the supraglottis and subglottis are less differentiated.

\section{Treatment of Carcinoma Larynx}

The treatment objective in early invasive carcinoma of the larynx is to obtain cure with laryngeal preservation and optimal voice quality with minimal morbidity, expense, and inconvenience. Early stage cancers are effectively treated with surgery or radiation. There are no randomized trials comparing surgery and radiation for the treatment of early stage larynx cancer. A Cochrane review compared the effectiveness of RT and surgery in early laryngeal cancer. It concluded that there was insufficient evidence to establish one modality as superior to the other. ${ }^{6}$ Conclusions regarding management is based on a comparison of nonrandomized studies, and both modalities are currently accepted as standard treatment options. ${ }^{7}$ In 2012 Yoo et al performed a systematic review regarding the treatment of $\mathrm{T} 1$ glottic tumours which incorporated one meta-analysis, 15 cohort studies, and two Cross sectional studies. They found no overall survival differences between surgery and radiation therapy.

\section{Early Vocal Cord Carcinoma (T1 and T2, Node Negative Disease)}

Irradiation is the initial treatment for $\mathrm{T} 1$ and $\mathrm{T} 2$ lesions, and surgery is reserved for salvage after radiation therapy failure. ${ }^{8}$ For $\mathrm{T}_{1}$ lesions, treated with primary $\mathrm{RT}$ the initial local control rates are in the range of $78 \%$ to $95 \%$, and the ultimate local control rates after surgical salvage of failures are in the range of $94 \%$ to $99 \% .{ }^{8,9}$ For $\mathrm{T}_{2}$ glottic tumours, RT is the preferred approach. Laser resection can be used for select tumours that are superficial and well localized. For $\mathrm{T}_{2}$ carcinoma of the glottis treated with primary RT, the initial local control rates are in the range of $67 \%$ to $88 \%$. After surgical salvage, the ultimate local control rates are in the range of $85 \%$ to $96 \% .8,9,10$

\section{Treatment of Early Supraglottic Cancer}

The prognosis for early stage supraglottic cancer is slightly worse than for early glottic cancer. All patients with early stage supraglottic larynx cancer should be treated with the intent to preserve the larynx. For early stage, favourable lesions, endoscopic laser resection, open-partial supraglottic laryngectomy, and radiation are all considered standard treatment options. ${ }^{11,12}$ Orus and colleagues showed slightly better initial local control with partial laryngectomy compared with radiation but, with salvage surgery, both resulted in ultimate local control of $90 \%$ for $\mathrm{T}_{1}$ - and $\mathrm{T}_{2}-\mathrm{N}_{0}$ supraglottic tumors. ${ }^{12}$

\section{Radiobiology of Altered Fractionation}

In principle, tumors should be treated for an overall treatment time that is as short as possible with acceptable acute morbidity, but with a dose per fraction that does not compromise late responding normal tissues, or total dose. The outcome of several large fractionation trials, mainly involving head and neck tumors, and particularly the CHART trial, have clearly demonstrated the importance of "acceleration" that is, shortening the overall treatment time, to improve local control. On the other hand, time taken for normal-tissue repair is about $6 \mathrm{hrs}$. This limits the strategy of using multiple treatments per day to maintain a large number of treatments in a short overall time. The alternative is a larger-dose per fractions. In hypofractionation there is increased tumour kill due to increased dose per fraction. Aside from tumor DNA damage, the extra effectiveness of hypofractionation may be due to its anti-angiogenic effect on micro-environment vasculature of tumour. But there are concerns about late tissue toxicity, cosmesis and local control rates. This is because the effective biologically equivalent dose (BED) used in this altered fractionation regime is actually less when compared to standard fractionation. For a well to moderately differentiated early glottis malignancy the BED will higher for the same dose and schedule compared to poorly differentiated tumours. This translates to higher local control for hypo fractionated regimens in better differentiated tumours. Approximately $90 \%$ early glottic carcinomas are of the well- or moderately-differentiated squamous type, implicating the clinical significance of hypofractionation in glottis malignancies.

\section{Local Control with Altered Fractionation}

Between December 1993 and December 2001, 189 patients with invasive, previously untreated, T1 squamous cell carcinoma of the true vocal cords were enrolled in a prospective randomized trial in the Department of Radiation Oncology, Osaka Medical Center for Cancer and Cardiovascular Diseases, Japan.The trial compared 2 Gy per fraction versus 2.25 Gy per fraction in the treatment of stage 1 glottic cancer.[13] Patients were randomly allocated to treatment arm A (2 Gy/fraction) or B (2.25 Gy/fraction. Of the 180 patients (170 men and 10 women), 144 had Stage T1a and 36 had Stage T1b. For minimal tumors, 60 Gy in 30 fractions within 6 weeks (Arm A1) was adopted as the conventional schedule and 56.25 Gy in 25 fractions within 5 weeks (Arm B1) for the 2.25-Gy arm. For larger than minimal tumors, 66 Gy in 33 fractions within 6.6 weeks (Arm A2) was used for the 2-Gy/fraction arm and 63 Gy in 28 fractions within 5.6 weeks (Arm) for the 2.25 -Gy/fraction arm. The dose was prescribed at the isocenter, and all patients received the scheduled dose. The 5 -year local control rate for the entire group was $86 \%$ (T1a, 83\%; T1b, 91\%; difference not significant), and the 5-year local control rates after radiotherapy were $76 \%$ for Arm A-1, 78\% for Arm A-2, 91\% for Arm B-1, and 92\% for Arm B-2. A significant difference was found in local control between Arm A (77\%) at 5 years Arm B (92\%). The 5-year overall survival rate was $88 \%$, 
without any significant difference between the two arms. This study established hypofractionation with 2.25 Gy as a standard fractionation for $\mathrm{T}_{1}$-No glottic cancer.13,14

\section{$\mathbf{T}_{2}$ Glottic Carcinoma}

For $\mathrm{T}_{2}$ carcinoma of the glottis treated with primary RT, the initial local control rates are in the range of $67 \%$ to $88 \%$. After surgical salvage, the ultimate local control rates are in the range of $85 \%$ to $96 \%$. The larynx is preserved in $71 \%$ to $88 \%$ of the patients irradiated. Many studies have shown that local control improves with hyper- or hypo fractionated radiation.[15,16] Garden and colleagues showed that more than 2 Gy per day improved local control whether it was delivered as more than 2 Gy per daily fraction or more than 2 Gy in smaller, twice-daily fractions ${ }^{[15,16]}$. In a retrospective study of T1N0 and T2N0 squamous cell carcinoma of glottic larynx treated with definitive RT by Bhishamjit S. Chera, et al in the Department of Radiation Oncology, University of Florida College of Medicine, Gainesville they analysed the effects of various parameters in local control-T stage (T1a vs. T1b vs. T2), anterior commissure invasion (yes vs. no), histologic differentiation (well, moderate, or not otherwise specified vs. poor), total dose ( $<63$ Gy or $>63 \mathrm{~Gy}$ ), fractionation (2.25 Gy daily vs. 1.2 Gy twice daily vs. $<2.25$ Gy daily), overall treatment time and beam energy (60 Co vs. other).They observed a 5 -year LC rate of more than $90 \%$ for $\mathrm{T} 1 \mathrm{a}$ and $\mathrm{T} 1 \mathrm{~b}$ tumors, whereas LC rate is $70 \%$ and $80 \%$ for T2 tumors. Factors that adversely affected LC were $\mathrm{T}$ stage, overall treatment time exceeding 41 days, and poorly differentiated histology. There was a concern of increase toxicity to altered fractionation schedule when treated with Cobalt machines. But in this study, there was no statistical difference between machines ${ }^{17}$

\section{Effects of Treatment on Normal Tissues}

Acute Effects of Radiation Therapy- Acute reactions during fractionated RT for carcinoma of the vocal cords are usually mild. It includes fatigue, hoarseness, sore throat, dysphagia, mucositis, and increased pigmentation of the skin in the radiation field 18 . They do not result in a treatment break. They are typically managed conservatively with topical anaesthetics, opioids, oral rinse solutions and antiinflammatory agents. They usually subside completely within 6 to 8 weeks after completion of treatment ${ }^{18}$. In the majority of patients, the voice returns to normal within a few months after treatment. The use of 2.25 Gy per fraction, did not increase the risk of acute skin or mucosal toxicity compared with 2 Gy per fraction.

Late Effects- Laryngeal oedema may persist after RT. The incidence of mild to moderate laryngeal oedema persisting for more than 3 months after RT is about $10 \%$ to $25 \% .18,20$, The incidence of severe laryngeal oedema is about $1.5 \%$ to $4.6 \%{ }^{20-23}$ The incidence of laryngeal oedema increases with greater total dose, field size, dose per fraction, and $\mathrm{T}$ stage of the lesion. When using doses up to 66 Gy in 2 Gy fractions or 63 Gy in 2.25 Gy fractions, Yamazaki and colleagues observed no severe late complications. ${ }^{24}$ Voice quality after radiation for larynx cancer is typically very good and superior to that achieved after larynx-preserving surgery. But up to $60 \%$ of patients experiences some decline in voice function. ${ }^{25}$ In up to $80 \%$ of patients voice will return to normal quality after radiation. But objective voice quality is almost always altered. Another common side effect of radiation to the head and neck region is hypothyroidism. $50 \%$ to $60 \%$ of patients who undergo thyroid-stimulating hormone (TSH) screening will have an elevated TSH level within 5 years of receiving 50 Gy to the low neck. When screening is not routinely used, the rate of hypothyroidism is around $30 \% .{ }^{25}$

We wanted to assess the locoregional control rate of early stage (T1/T2) glottic carcinoma larynx and toxicity outcome of patients treated with hypo fractionated radical radiotherapy (63 Gy/28\#). We also wanted to compare the toxicity profile and outcome between Cobalt and Linac Machines treatment.

\section{METHODS}

A total of 59 patients, newly diagnosed with stage I and II squamous cell carcinoma of glottic larynx who attended the OP clinic of Radiation Oncology department of Kottayam Medical College from November 2016 to November 2017 who were intended to radically treat with a hypo fractionated regimen of radiotherapy were analysed for local control and toxicity.

\section{Statistical Analysis}

Data analysis was be done with the help of Excel 2010 and SPSS 16 statistical software. Remission rates, toxicity grades and survival rates will be entered in Excel 2010 work sheet for each variable. The quantitative variables were analysed using chi square test. Local failure is defined by residual disease or recurrence at primary site alone (Glottis larynx). Loco regional failure is defined by residual disease or recurrence at all sub sites of larynx and regional lymph nodes. The highest toxicity during any cycle was considered as toxicity grade of that patient. The toxicities occurred in Linear accelerator and Cobalt 60 machines were compared with cross tabulation by Mann Whitney U test. Survivals in 59 patients were assessed by Kaplan Meir survival plot.

\section{RESULTS}

A total of 59 patients, newly diagnosed with stage I and II squamous cell carcinoma of glottic larynx who attended the OP clinic of Radiation Oncology department of Kottayam Medical College from November 2016 to November 2017 who were intended to radically treat with a hypo fractionated regimen of radiotherapy were analysed. Patient related characteristics are listed in (Table 1.)

Males are more commonly affected by carcinoma larynx (994\%) and smoking is the major etiological factor (93\%). As the pack years increased the chance of laryngeal cancer also rises. (The trends are shown in the bar diagrams.) ENT evaluation showed a growth in one vocal cord in $42(71.2 \%)$ patients and growth in both vocal cords in $17(28.8 \%)$ patients. In $22(37.3 \%)$ patients there was anterior commissure involvement. In $9(15.3 \%)$ patients there was impaired vocal cord mobility. $100 \%$ of the patients are having squamous cell carcinoma. Most of the patients are from Dr. 
Kuppuswamy Lower middle income (57\%) and upper middle (30\%) group.

\begin{tabular}{|c|c|c|}
\hline Age & \multicolumn{2}{|c|}{ Frequency } \\
\hline Mean & \multicolumn{2}{|c|}{59.59} \\
\hline SD & \multicolumn{2}{|c|}{7.67} \\
\hline Min & \multicolumn{2}{|c|}{42} \\
\hline $\max$ & \multicolumn{2}{|c|}{75} \\
\hline Gender & Frequency & $\%$ \\
\hline Male & 56 & 94.9 \\
\hline Female & 3 & 5.1 \\
\hline Tobacco & Frequency & $\%$ \\
\hline Yes & 55 & 93.2 \\
\hline no & 4 & 6.8 \\
\hline Pack years (Yrs.) & Frequency & $\%$ \\
\hline $0(0-10)$ & 6 & 10.2 \\
\hline $1(10-20)$ & 4 & 6.8 \\
\hline $2(20-30)$ & 9 & 15.3 \\
\hline $3(30-40)$ & 15 & 25.4 \\
\hline $4(40-50)$ & 20 & 33.9 \\
\hline $5(50-60)$ & 5 & 8.5 \\
\hline Alcohol & Frequency & $\%$ \\
\hline Yes & 4 & 6.8 \\
\hline No & 55 & 93.2 \\
\hline Laryngitis & Frequency & $\%$ \\
\hline Yes & 3 & 5.1 \\
\hline No & 56 & 94.9 \\
\hline Hoarseness of voice Duration & \multicolumn{2}{|c|}{ Frequency } \\
\hline Median & \multicolumn{2}{|c|}{4} \\
\hline $\mathrm{IQR}$ & \multicolumn{2}{|c|}{1.5} \\
\hline Min & \multicolumn{2}{|c|}{1} \\
\hline Max & \multicolumn{2}{|c|}{48} \\
\hline ECOG performance status & Frequency & $\%$ \\
\hline 0.1 & 58 & 98.3 \\
\hline 2 & 1 & 1.7 \\
\hline Vocal cord Involvement & Frequency & $\%$ \\
\hline T1 a & 42 & 71.2 \\
\hline T1 b & 17 & 28.8 \\
\hline Anterior commissure & Frequency & $\%$ \\
\hline Present & 22 & 37.3 \\
\hline Absent & 37 & 62.7 \\
\hline Vocal cord mobility & Frequency & $\%$ \\
\hline Present & 50 & 84.7 \\
\hline Absent & 9 & 15.3 \\
\hline Histopathology & Frequency & $\%$ \\
\hline Well differentiated & 36 & 61 \\
\hline Moderately differentiated & 23 & 39 \\
\hline Pallor & Frequency & $\%$ \\
\hline Yes & 3 & 5.1 \\
\hline No & 56 & 94.9 \\
\hline Clubbing & Frequency & $\%$ \\
\hline Yes & 26 & 44.1 \\
\hline No & 33 & 55.9 \\
\hline Table 1 & t Characte & \\
\hline
\end{tabular}

\begin{tabular}{|c|c|c|c|c|}
\hline Machine & \multicolumn{3}{|c|}{ Frequency } & Percent \\
\hline Theratron & \multicolumn{3}{|c|}{31} & 52.5 \\
\hline Clinac & \multicolumn{3}{|c|}{28} & 47.5 \\
\hline Total & \multicolumn{3}{|c|}{59} & $100 \%$ \\
\hline Local control & \multicolumn{3}{|c|}{ Frequency } & Percentag \\
\hline Achieved & \multicolumn{3}{|c|}{55} & 93.2 \\
\hline Not Achieved & \multicolumn{3}{|c|}{4} & 6.8 \\
\hline Local Control and T Stage & \multicolumn{2}{|c|}{ LC yes } & LC no & p-value \\
\hline $\mathrm{T} 1$ & \multicolumn{2}{|c|}{47} & 3 & .574 \\
\hline $\mathrm{T} 2$ & \multirow{2}{*}{\multicolumn{2}{|c|}{8}} & 1 & \\
\hline Machine and Local Control & & LC yes & LC no & p-value \\
\hline COBALT -60 & \multirow{2}{*}{\multicolumn{2}{|c|}{$\frac{29}{26}$}} & 2 & \multirow{2}{*}{0.916} \\
\hline LINAC & & & 2 & \\
\hline Treatment Duration & Median & Iqr & Median-Diff & p-Value \\
\hline Cobalt 60 & 4 & 1.5 & .50 & \\
\hline LINAC & 4.50 & 2.25 & & 0.48 \\
\hline Mucositis Grade & \multicolumn{2}{|c|}{ Frequency } & \multicolumn{2}{|c|}{ Percent } \\
\hline Grade 1 & \multicolumn{2}{|c|}{14} & \multicolumn{2}{|c|}{23.7} \\
\hline Grade 2 & \multicolumn{2}{|c|}{36} & \multicolumn{2}{|c|}{61.0} \\
\hline Grade 3 & \multicolumn{2}{|c|}{9} & \multicolumn{2}{|c|}{15.3} \\
\hline Total & \multicolumn{2}{|c|}{59} & \multicolumn{2}{|c|}{100.0} \\
\hline Duration of treatment & \multicolumn{4}{|c|}{ Weeks } \\
\hline Min & \multicolumn{4}{|c|}{5.5} \\
\hline $\operatorname{Max}$ & \multirow{2}{*}{\multicolumn{4}{|c|}{$\begin{array}{c}7 \\
5.89\end{array}$}} \\
\hline Mean & & & & \\
\hline SD & \multicolumn{4}{|c|}{$\begin{array}{l}5.89 \\
.299\end{array}$} \\
\hline \multicolumn{5}{|c|}{ Table 2. Treatment Related Factors } \\
\hline
\end{tabular}

\section{Treatment Related Factors}

All patients were treated with radical hypo fractionated radiotherapy of $63 \mathrm{~Gy} / 28 \#$. The treatment duration ranged from 5.5 to 6.4 weeks with a mean duration of 5.89 weeks. $31(52.5 \%)$ patients were treated in Theratron $780 \mathrm{C}$ cobalt machine and $28(47.5 \%)$ patients in Varian CLINAC machine according to the availability of treatment slots. The mean field height was $7.3 \mathrm{~cm}$ and width was $7.5 \mathrm{~cm}$. Details summarised in (Table 2).

\section{Toxicities Assessment}

Treatment related toxicities are assessed with CTCAE version 4. Grade 3 dermatitis was noted in $2(3.4 \%)$ patients during $5^{\text {th }}$ week and in 11 patients $(18.6 \%)$ during $6^{\text {th }}$ week The 1 year local control rate was 93. $2 \%$. The mean time for local conntrol was 11.6 months (with $95 \%$ confidence interval). Pain was present for 55 patients (93.2\%) and is gradually incresed with accumulatin of radiation dose and fractions and by $6^{\text {th }}$ week,45(76.3\%) patients had grade 1 pain and 10 patients devoloped grade 2 pain. Anorexia is present for 32 patients (54.2\%) and is grade 1 only.32 patients (54.2\%) had ryles tube feeding at some point during radiotherapy and there was no significant weight loss associated with radiation. 6 patients had grade 1 vomiting (10.2\%) and is relieved with supportive care and is statistically not significant. Mucositis was the major toxicity limiting radiation treatment and was associated with pain. $100 \%$ patients has mucositis by $2^{\text {nd }}$ weeek. 55 had grade 1 and 4 had grade 2 mucositis by $2^{\text {nd }}$ week. Mucositis was evaluated in detail with respect to the machine used for radiation treatment and the impact was assessed. The results are given below.

\section{Association between Treatment Machine Used and Grade 2 and 3 Mucositis}

Mucositis is the major treatment limits toxicity associated with pain and anorexia and resultant weight loss. This can lead to radiation treatment breaks which can be detrimental on disease outcome. Grade 1 mucositis can be supported without any treatment interruption. But grade 2/ grade 3 mucositis can affect locoregional control and survival by producing treatment breaks. Hence it was assessed in detail as given below.

\begin{tabular}{|c|c|c|c|c|c|c|}
\hline \multicolumn{7}{|c|}{ Cross Tabulation Week 3} \\
\hline \multicolumn{7}{|c|}{ Mucositis } \\
\hline & achine & Grade 1 & Grade 2 & Total & \multirow{3}{*}{\multicolumn{2}{|c|}{ p-value }} \\
\hline \multicolumn{2}{|c|}{ Cobalt-60 Linac } & 16 & 15 & 31 & & \\
\hline \multirow{2}{*}{\multicolumn{2}{|c|}{$\begin{array}{l}\text { Linac } \\
\text { Total }\end{array}$}} & 25 & 3 & 28 & & \\
\hline & & 41 & 18 & 59 & & \\
\hline \multicolumn{7}{|c|}{ Table 3. Mucositis Week 3} \\
\hline \multicolumn{7}{|c|}{ Cross Tabulation week 6} \\
\hline \multicolumn{7}{|c|}{ Mucositis } \\
\hline \multirow{3}{*}{ Machine } & & \begin{tabular}{l|l} 
Grade 1 \\
\end{tabular} & Grade 2 & Grade 3 & Total & p-value \\
\hline & Cobalt-60 & 3 & 22 & 6 & 31 & \multirow{2}{*}{0.027} \\
\hline & Linac & 11 & 14 & 3 & 28 & \\
\hline Total & & 14 & 36 & 9 & 59 & \\
\hline \multicolumn{7}{|c|}{ Table 3a. Mucositis Week 6} \\
\hline
\end{tabular}

This cross tabulation shows a statistically significant $(p=0.027)$ association between the machine used and the grade $2 / 3$ mucositis developed at week 6.22 patients developed grade 2 mucositis with Cobalt 60 machine but only 14 patients in Linear accelerator. The grade 3 toxicity is reduced by $50 \%$ with radiation treatment taken in linear 
accelerator. The mucositis is progressively increased from week 2 of radiation for patients treated with Cobalt 60 Machine. This is associated with significant increase in pain and anorexia but can be managed by supportive care.

\section{Association between Machine Used and Grade 2 \& 3 Dermatitis}

A weekly assessment of dermatitis between the machine is being done, but it was not statistically significant till week 3 . In week 4, the assessment revealed a trend towards improved incidence of dermatitis in patients treated with linear accelerator $(p=0.511)$ than Cobalt 60 machine but it was not statistically significant. This effect persisted in week 5 and 6.

\begin{tabular}{|c|c|c|c|c|c|c|}
\hline \multicolumn{6}{|c|}{ Machine * Dermatitis at Wks. 6 Cross Tabulation } & \multirow{3}{*}{ p-value } \\
\hline \multirow{2}{*}{ Count } & \multicolumn{4}{|c|}{ Dermwk6 } & \multirow{2}{*}{ Total } & \\
\hline & & 1 & 2 & 3 & & \\
\hline \multirow{2}{*}{ Machine } & Co 60 & 3 & 23 & 5 & 31 & \multirow{2}{*}{0.511} \\
\hline & Linac & 5 & 17 & 6 & 28 & \\
\hline \multicolumn{2}{|c|}{ Total } & 8 & 40 & 11 & 59 & \\
\hline
\end{tabular}

\section{Locoregional Control and Survival}

Local control was achieved in $93.2 \%$ patients at the end of study period by Hypo fractionated radiotherapy protocol. 4 patients developed loco regional failure and 2 patients died of the disease. The overall survival was 96.61 at the assessment period of the study.

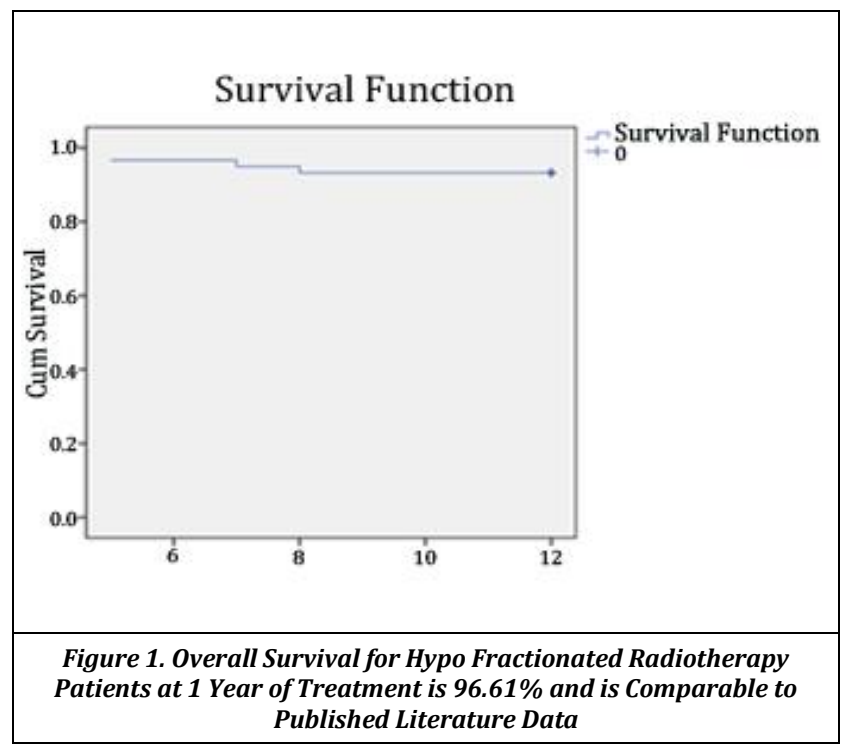

\section{DISCUSSION}

Cancer of the larynx is the most common malignancy of upper aerodigestive tract. While in early stage glottic malignancy surgery and radiotherapy has equal results in terms of cure rates, radiotherapy has the advantage of preserving voice quality. ${ }^{26-30} \mathrm{~A}$ useful voice is preserved in 80 to $95 \%$ of patients who are treated with radiation and 80 to $90 \%$ are reported to have good to excellent voice quality. A standard course of radiation for early glottic cancer consists of 2 gray per fraction, once a day, 5 days a week, to a total dose of 70 gray in 35 fractions during 7 weeks. In this study a hypo fractionated schedule of radiation therapy was used- dose of 2.25 gray per fraction, once a day, 5 days a week to a total dose of 63 gray in 28 fractions during 6 weeks. 59 patients with biopsy proven early squamous cell carcinoma of glottic larynx who attended the outpatient clinic of Department of Radiation Oncology, Medical College Kottayam were included in the study.

\section{Patient Related Factors}

The mean age of patients included in the study was 60 years with ages ranging from 42 to 75 years. $94.9 \%$ were males and $5.1 \%$ females. This higher incidence in males is in accordance with the data from national cancer registry and also the international data of incidence. ${ }^{2,3}$ The higher incidence in males and the strongest etiological factor of smoking has a positive association. Majority of the patients (93.2\%) were smokers with pack years of 30 to 40 years. $93.2 \%$ gave a history of chronic alcohol intake. Studies have proven that $75 \%$ of laryngeal carcinomas are attributable to cigarette smoking and alcoholism. The excess risk declines when smoking is ceased and becomes negligible after 20 years. In our study the patients were current smokers or had recently stopped smoking after the onset of symptoms. There was no significant association between the smoking status of the patient and local or locoregional control in our study. Similar results were observed in the study conducted by Lee et al. ${ }^{30}$ Hoarseness is the most common symptom in early vocal cord cancer and is the main reason why glottic malignancies are usually diagnosed at an early stage. ${ }^{8}$ ENT evaluation showed a growth in one vocal cord (T1a) in $42(71.2 \%)$ patients and growth in both vocal cords (T1b) in $17(28.8 \%)$ patients. In $22(37.3 \%)$ patients there was anterior commissure involvement. In $9(15.3 \%)$ patients there was impaired vocal cord mobility. (T2). In the study conducted by Lee et al, anterior commissure involvement was associated with decreased local control for T1 lesions. For T2 lesions local control decreased with impaired vocal cord mobility and subglottic extension. In this study no significant association could be made between the tumour extend and locoregional failure. This is probably due to the small sample size and less number of events of locoregional failure. ${ }^{30}$

\section{Treatment Related Factors}

In our study patients were treated in Theratron 780C cobalt 60 machine or Varian Clinac machine according to the availability of treatment slots. 31(52.5\%) patients were treated in Theratron 780C cobalt machine and 28 (47.5\%) patients in Varian CLINAC machine. There was no significant association between the local failure rate and machine/energy used in treatment. The findings of the prospective trial by Le et al[30] gave a similar result. In the study beam energy (cobalt 60 vs $6 \mathrm{MV}$ vs $4 \mathrm{MV}$ ) was not significantly associated with the local control following radiotherapy. The treatment duration ranged from 5.5 to 6.4 weeks with a mean duration of 5.89 weeks. This range was owing to delays due to personal reasons and machine delays in 4 cases. In this study no significant association was established between locoregional control and treatment duration. 


\section{Acute Adverse Events}

An objective of the study was to assess the treatment related toxicities and their grading according to CTCAE $v$ 4. Pain, mucositis, anorexia, vomiting and dermatitis were evaluated.

During the $4^{\text {th }}$ week of treatment $50(84.7 \%)$ of patients had grade 2 skin toxicity. By $5^{\text {th }}$ week $38(64.4 \%)$ patients had grade 2 and $2(3.4 \%)$ patients had grade 3 dermatitis. This progressed to grade 2 toxicity in $40(67.8 \%)$ patients and grade 3 toxicity in $11(18.6 \%)$ patients during the $6^{\text {th }}$ week. $10(16.9 \%)$ patients experienced grade 2 pain during the $6^{\text {th }}$ week of treatment. There was no documented grade 2 or higher anorexia during the radiation course. 4 (6.8\%) patients experienced grade 2 mucositis during the $2^{\text {nd }}$ week of treatment. By $6^{\text {th }}$ week 36 patients $(61 \%)$ had grade 2 mucositis and 9 (15.3\%) patients had grade 3 mucositis. There was no grade 4 mucositis recorded in the study. There was no grade 2 or higher vomiting in any of the patients.

Majority of the patients in the study had grade $1-2$ dysphagia, grade 2- 3 dermatitis, grade 2-3 laryngeal mucositis. None of the patients in the study had any grade 4 toxicities. The mean time of recovery from dermatitis was 2.5 weeks with no statistically significant difference in recovery time between the patients treated in the two machines. The recovery from hoarseness due to laryngeal mucositis and oedema began by a mean duration of 4 months. There was no persistent laryngeal oedema requiring tracheostomy in any of the patients.

In this study there was statistically significant difference between the machine in which patient received treatment and the incidence of grade 2 or higher dermatitis and laryngeal mucositis. During week 2, 3 and 6 of treatment there was higher grade 2 and 3 laryngeal mucositis among patients treated in cobalt 60. During week 4 there was significantly higher incidence of grade 2 and 3 dermatitis among patients treated in cobalt 60 machine. This finding suggests that there is a trend towards better toxicity profile in terms of dermatitis and laryngeal mucositis in patients treated in Linac though there is no difference in local control between the two beam energies used in treatment. This result is contradictory to the published data that cobalt 60 is non inferior to linear accelerator in terms of acute skin toxicity. This finding needs further validation in larger trials.

The primary objective of our study to find out the local and loco regional control at a period of one year. In our study local control was achieved in $55(93.2 \%)$ patients at 1 year. There was loco regional failure in $4(6.8 \%)$ patients. These results were similar to the study conducted by Yamasaki et al [29] where the 5 year loco regional control following primary treatment was $92 \%$ in the hypo fractionated RT arm and 77\% in the conventional RT arm. The results of our hypo fractionated regimen is thus comparable to the historical data of similar studies using a hypo fractionated dose of radiation and superior to the conventional fractionation in terms of local control.

From the point of view of health economics, the hypo fractionated schedule is advantageous compared to conventional schedule, because the shorter overall treatment time reduces the socioeconomic burden, both for patients and institutions especially in less privileged countries. Patients benefit from the reduced costs, treatment time and early resolution of acute toxicities of radiation. The institutions can maintain the machine and human resources required to meet the increasing demand for radiotherapy.

\section{CONCLUSIONS}

Hypo fractionated external beam radiotherapy of 63 Gy/28\# for early stage glottic carcinoma has comparable outcome to conventionally fractionated regimens and is convenient due to shorter overall treatment time which reduces the socioeconomic burden, both for patients and institutions. However, this study suggests an improved toxicity outcome in hypo fractionated radiotherapy for patients treated with linear accelerator compared to cobalt 60 Machine.

\section{REFERENCES}

[1] Jemal A, Siegel R, Ward E, et al. Cancer statistics, 2008. CA Cancer J Clin 2008;58(2):71-96.

[2] Three-year Report of Population Based Cancer Registries 2009-2011. Bangalore: National Cancer Registry Program (ICMR), 2013.

[3] Hashibe M, Brennan P, Benhamou S, et al. Alcohol drinking in never users of tobacco, cigarette smoking in never drinkers, and the risk of head and neck cancer: pooled analysis in the International Head and Neck Cancer Epidemiology Consortium. J Natl Cancer Inst 2007;99(10):777-89.

[4] Muscat JE, Wynder EL. Tobacco, alcohol, asbestos and occupational risk factors for laryngeal cancer. Cancer 1992;69(9):2244-51.

[5] Ward PH, Hanson DG. Reflux as an etiological factor of carcinoma of the laryngopharynx. Laryngoscope 1988;98(11):1195-9.

[6] Mlynarek A, Kost K, Gesser R. Radiotherapy versus surgery for early T1-T2 glottic carcinoma. J Otolaryngol 2006;35(6):413-9.

[7] Brandbo K, Benninger MS. Laser resection of T1a glottic carcinomas: results and postoperative voice quality. Acta Otolaryngol 2004;124(8):976-9.

[8] Mendenhall WM, Morris CG, Amdur RJ, et al. Parameters that predict local control following definitive radiotherapy for squamous cell carcinoma of the head and neck. Head Neck 2003;25(7):535-42.

[9] Mendenhall WM, Amdur RJ, Morris CG, et al. T1-T2N0 squamous cell carcinoma of the glottic larynx treated with radiation therapy. J Clin Oncol 2001;19(20):402936.

[10] Mendenhall WM, Werning JW, Hinerman RW, et al. Management of T1-T2 glottic carcinomas. Cancer 2004;100(9):1786-92.

[11] O'Sullivan B, Mackillop W, Gilbert R, et al. Controversies in the management of laryngeal cancer: results of an international survey of patterns of care. Radiother Oncol 1994;31(1):23-32.

[12] Orus C, Leon X, Vega $M$, et al. Initial treatment of the early stages (I, II) of supraglottic squamous cell carcinoma: partial laryngectomy versus radiotherapy. Eur Arch Otorhinolaryngol 2000;257(9):512-6. 
[13] Yamazaki H, Nishiyama K, Tanaka E, et al. Radiotherapy for early glottic carcinoma (T1N0M0): results of prospective randomized study of radiation fraction size and overall treatment time. Int J Radiat Oncol Biol Phys 2006;64(1):77-82.

[14] Mendenhall WM, Parsons JT, Million RR, et al. T1-T2 squamous cell carcinoma of the glottic larynx treated with radiation therapy: relationship of dosefractionation factors to local control and complications. Int J Radiat Oncol Biol Phys 1988;15(6):1267-73.

[15] Haugen H, Johansson KA, Mercke C. Hyperfractionatedaccelerated or conventionally fractionated radiotherapy for early glottic cancer. Int J Radiat Oncol Biol Phys 2002;52(1):109-19.

[16] Garden AS, Forster K, Wong PF, et al. Results of radiotherapy for T2N0 glottic carcinoma: does the "2" stand for twice-daily treatment? Int J Radiat Oncol Biol Phys 2003;55(2):322-8.

[17] Chera BS, Amdur RJ, Morris CG, et al. T1N0 to T2 N0 Squamous cell carcinoma of the glotic larynx treated with definitive radiation therapy. Int J Radiat Oncol Biol Phys 2010;78(2):461-6.

[18] Caudell JJ, Schaner PE, Meredith RF, et al. Factors associated with long-term dysphagia after definitive radiotherapy for locally advanced head-and-neck cancer. Int J Radiat Oncol Biol Phys 2009;73(2):410-5.

[19] Dinshaw KA, Sharma V, Agarwal JP, et al. Radiation therapy in T1-T2 glottic carcinoma: influence of various treatment parameters on local control/complications. Int J Radiat Oncol Biol Phys 2000;48(3):723-35.

[20] Mendenhall WM, Riggs CE, Amdur RJ, et al. Altered fractionation and/or adjuvant chemotherapy in definitive irradiation of squamous cell carcinoma of the head and neck. Laryngoscope 2003;113(3):546-51.

[21] Woodhouse RJ, Quivey JM, Fu KK, et al. Treatment of carcinoma of the vocal cord. A review of 20 years of experience. Laryngoscope 1981;91(7):1155-62.
[22] Howell-Burke D, Peters LJ, Goepfert H, et al. T2 glottic cancer. Recurrence, salvage and survival after definitive radiotherapy. Arch Otolaryngol Head Neck 1990;116(7):830-5.

[23] Amornmarn R, Prempree T, Viravathana T, et al. A therapeutic approach to early vocal cord carcinoma. Acta Radiol Oncol 1985;24(4):321-5.

[24] Mendenhall WM, Parsons JT, Brant TA, et al. Is elective neck treatment indicated for T2N0 squamous cell carcinoma of the glottic larynx? Radiother Oncol 1989;14(3):199-202.

[25] Norris AA, Amdur RJ, Morris CG, et al. Hypothyroidism when the thyroid is included only in the low neck field during head and neck radiotherapy. Am J Clin Oncol Cancer Clin Trials 2006;29(5):442-5.

[26] Epstein BE, Lee DJ, Kashima H, et al. Stage T1 glottic carcinoma: results of radiation therapy or laser excision. Radiology 1990;175(2):567-70.

[27] Jones AS, Fish B, Fenton JE, et al. The treatment of early laryngeal cancers (T1-T2 N0): surgery or irradiation? Head Neck 2004;26(2):127-35.

[28] Loughran S, Calder N, MacGregor FB, et al. Quality of life and voice following endoscopic resection or radiotherapy for early glottic cancer. Clin Otolaryngol 2005;30(1):42-7.

[29] Goor KM, Peeters AJ, Mahieu HF, et al. Cordectomy by $\mathrm{CO} 2$ laser or radiotherapy for small T1a glottic carcinomas: costs, local control, survival, quality of life, and voice quality. Head Neck 2007;29(2):128-36.

[30] Le QT, Fu KK, Kroll S, et al. Influence of fraction size, total dose and overall time on local control of T1-T2 glottic carcinoma. Int J Radiat Oncol Biol Phys 1997;39(1):11526. 